\title{
The CpxR regulates type VI secretion system 2 expression and facilitates the interbacterial competition activity and virulence of avian pathogenic Escherichia coli
}

\author{
Zhengfei Yi, Dong Wang, Suhua Xin, Dongliang Zhou, Tao Li, Mingxing Tian, Jingjing Qi, Chan Ding, \\ Shaohui Wang ${ }^{*}$ and Shengqing Yu*
}

\begin{abstract}
Systemic infections caused by avian pathogenic Escherichia coli (APEC) are economically devastating to poultry industries worldwide and are also potentially threatening to human health. Pathogens must be able to precisely modulate gene expression to facilitate their survival and the successful infection. The Cpx two-component signal transduction system (TCS) regulates surface structure assembly and virulence factors implicated in Gram-negative bacterial pathogenesis. However, the roles of the CpxTCS in bacterial fitness and pathogenesis during APEC infection are not completely understood. Here, we show that the CpxTCS response regulator CpxR is critical to the survival and virulence of APEC. Inactivation of $c p x R$ leads to significant defects in the interbacterial competition activity, invasion and survival of APEC in vitro and in vivo. Moreover, activation of CpxR positive regulates the expression of the APEC type $\mathrm{VI}$ secretion system 2 (T6SS2). Further investigations revealed that phosphorylated CpxR directly bound to the T6SS2 hсp2B promoter region. Taken together, our results demonstrated that CpxR contributes to the pathogensis of APEC at least through directly regulating the expression and function of T6SS2. This study broadens understanding of the regulatory effect of CpxTCS, thus elucidating the mechanisms through which Cpx TCS involved in bacterial virulence.
\end{abstract}

\section{Introduction}

Systemic infections caused by avian pathogenic Escherichia coli (APEC) are economically devastating to poultry industries worldwide. APEC shares a broad range of virulence factors with human extraintestinal pathogenic E. coli (ExPEC), including uropathogenic E. coli (UPEC) and neonatal meningitis E. coli (NMEC). Moreover, these ExPEC strains can cause cross infections, thus indicating that APEC may be a potential virulence gene reservoir for UPEC and NMEC [1-4]. APEC initially infects poultry via the respiratory tract, then spreads systemically throughout the entire body. Pathogens use several common strategies to increase fitness and facilitate survival and systemic infections $[5,6]$. The conserved type VI

*Correspondence: shwang0827@126.com; yus@shvri.ac.cn Shanghai Veterinary Research Institute, Chinese Academy of Agricultural Sciences, Shanghai 200241, China secretion system (T6SS), present in more than one-fourth of Gram-negative pathogens, is used to deliver effector proteins into eukaryotic or prokaryotic cells. These effectors have roles in a broad variety of functions, including interbacterial competition, stress sensing, biofilm formation and virulence [7-13]. Three distinct T6SSs have been identified in APEC genomes. Among them, the APEC T6SS2, similar to NMEC T6SS, is responsible for the binding and invasion to host cells, survival, interbacterial competition and pathogenesis of APEC and NMEC $[11,14-16]$. As contact-dependent pathways, the T6SSs are tightly regulated by various regulatory mechanisms to ensure successful bacterial infection [17].

When detecting new environmental cues or stresses, bacteria must precisely modulate gene expression to facilitate their survival through complex regulatory networks. The two-component signal transduction systems (TCSs) consist of a histidine kinase sensor 
in the bacterial plasma membrane and a cytoplasmic response regulator that allows bacteria to cope with changes in the environment $[18,19]$. The Cpx TCS of E. coli consists of three proteins, CpxA, CpxR, and CpxP, which mainly mediate the detection of and adaptation to envelope stresses [20, 21]. Many environmental cues leading to $\mathrm{Cpx}$ TCS activation have been identified, including envelope protein misfolding, overexpression of the pilus and alkaline $\mathrm{pH}$. In addition, adhesion to hydrophobic surfaces activates the Cpx TCS in an NlpE-dependent manner [22-24]. After an environmental signal is received, the kinase histidine CpxA undergoes autophosphorylation and phosphorylates the response regulator CpxR. Then phosphorylated CpxR binds its regulon and functions as a transcriptional regulator. The third component of the Cpx system is CpxP, a small periplasmic protein thought to negatively regulate the Cpx TCS by binding CpxA and maintaining it in an inactive state [23-25].

The Cpx TCS affects bacterial virulence through regulating the expression of virulence genes involved in envelope stress relief, biofilm formation, adherence, motility and secretion systems (type III, type IV and type VI) [26-36]. The activation of Cpx TCS in Shigella sonnei, Legionella pneumophila, Xenorhabdus nematophila and Yersinia pestis results in increased expression of virulence genes and contributes to pathogenesis $[26,30,32,33,37,38]$. The Cpx TCS also regulates T6SS expression, and affects the virulence of Citrobacter rodentium [31, 36]. However, constitutive activation of the Cpx TCS results in downregulation of virulence genes and attenuated virulence of Haemophilus ducreyi $[39,40]$. In pathogenic E. coli, the Cpx TCS has been implicated in the regulation of pili and the type III secretion system and is responsible for virulence $[27,28,41-44]$. The Cpx TCS has been shown to be involved in the adherence, invasiveness and biofilm formation of APEC through controlling the orientation of the type 1 fimbriae OFF-ON switch. However, the roles of Cpx TCS in the fitness and pathogenesis of APEC during in vivo infection are not completely understood. In this study, we demonstrated that $c p x R$ deletion leads to substantial defects in interbacterial competition activity, invasion and survival, and attenuates the virulence of APEC in vitro and in vivo. Moreover, we provide evidence that CpxR positively regulates the expression and function of APEC T6SS2, which may contribute to the systemic infection and pathogenesis of APEC. This study broadens understanding of the regulatory effect of Cpx TCS, thereby elucidating the mechanisms through which Cpx TCS contributes to virulence.

\section{Materials and methods}

\section{Bacterial strains, plasmids and growth conditions}

The strains and plasmids used in this study are listed in Table 1. The APEC strain DE719 (O2:K1) causes severe colibacillosis symptoms and high mortality in ducks and mice [14]. Bacteria were grown routinely in Luria-Bertani (LB) medium at $37{ }^{\circ} \mathrm{C}$ with aeration. When necessary, the antibiotic ampicillin (Amp; $100 \mu \mathrm{g} / \mathrm{mL}$ ) or chloramphenicol $(\mathrm{Cm} ; 30 \mu \mathrm{g} / \mathrm{mL})$ was supplemented in the medium.

\section{Construction of mutant and complemented strains}

The $c p x R$ gene deletion mutant was constructed using the Lambda Red recombinase system and appropriate primers (Table 2), as described previously [45] with some modifications. For complementation, the open reading frame and putative promoter of $c p x R$ were subcloned into the low-copy plasmid pSTV28 (Takara, Dalian, China), which was then transformed into the mutant strain. The obtained mutant and complemented strains were validated by PCR and sequencing. For NlpE overexpression, the $n l p E$ gene was cloned into the plasmid pBAD/MycHis (Invitrogen, Carlsbad, CA, USA) and transformed into the APEC wild-type and mutant strains. To induce $n l p E$ expression, we supplemented bacterial cultures with L-arabinose and performed appropriate antibiotic selection.

\section{Experimental animal infection}

To determine the effect of CpxR on APEC virulence, we intramuscularly injected groups of eight 7-day-old ducks with bacterial suspensions containing $10^{5}$ Colony-Forming Units (CFUs). The number of CFUs in the injected inoculum was confirmed by plating on LB agar. Ducks inoculated with PBS were used as negative controls. Mortality was monitored daily until 7 days after infection.

The bacterial survival and competitive assays in vivo were measured as described previously [14, 46, 47]. Briefly, 7-day-old ducks were infected intratracheally with $10^{8} \mathrm{CFU}$ bacterial suspensions with wild-type or mutant strains. Bacterial mixtures with equal amounts of wild-type and mutant strains were inoculated into ducks for the competitive assays. After $24 \mathrm{~h}$, the ducks were euthanized and dissected, and the lung, liver and spleen were collected, weighed and homogenized. Serial dilutions of the homogenates were plated onto LB agar with or without chloramphenicol to distinguish the mutant strain or total bacterial loads. The competitive index (CI) was calculated for the mutant by dividing the output ratio (mutant/wild-type) by the input ratio (mutant/ wild-type). 
Table 1 Bacterial strains and plasmids used in this study

\begin{tabular}{|c|c|c|}
\hline Strains or plasmids & Characteristics & References \\
\hline \multicolumn{3}{|l|}{ Strains } \\
\hline DE719 & $\mathrm{O} 2: \mathrm{K} 1$ & [14] \\
\hline$\Delta c p x R$ & cpxR gene deletion mutant in DE719 & This study \\
\hline$C \Delta c p x R$ & $\Delta c p x R$ with plasmid pSTV28-cpxR & This study \\
\hline DH5a-pRCL & DH5a with plasmid pRCL & This study \\
\hline DH5a-pRCL-P ${ }_{h c p 2 B}$ & DH5a with plasmid pRCL-P $P_{h c p 2 B}$ & This study \\
\hline DE719-pBAD & DE719 with plasmid pBAD/Myc-His & This study \\
\hline DE719-pnlpE & DE719 with plasmid pBAD-nlpE & This study \\
\hline$\triangle c p x R-p B A D$ & $\triangle c p x R$ with plasmid $\mathrm{pBAD} / \mathrm{Myc}-\mathrm{His}$ & This study \\
\hline$\Delta c p x R-p n l p E$ & $\triangle c p x R$ with plasmid pBAD-nlpE & This study \\
\hline $\mathrm{DH} 5 \mathrm{a}$ & $\mathrm{F}-, \triangle($ lacZYA-argF)U169, recA1, endA1, hsdR17(rk-, mk+), phoA, supE44, $\lambda-$ & TIANGEN \\
\hline BL21 (DE3) & $\mathrm{F}-, \mathrm{ompT}, \mathrm{hsdS}\left(\mathrm{r}_{B}^{-} m_{B}^{-}\right) \mathrm{gal}, \mathrm{dcm}(\mathrm{DE} 3)$ & TIANGEN \\
\hline \multicolumn{3}{|l|}{ Plasmids } \\
\hline pET28a $(+)$ & Kan, F1 origin, His tag & Novagen \\
\hline pET28a-cpxR & pET28a $(+)$ carrying cpxR gene & This study \\
\hline pSTV28 & $\mathrm{Cm}, \mathrm{p} 15 \mathrm{~A}$ origin & Takara \\
\hline pSTV28-cpxR & pSTV28 derivative harboring cpxR gene & This study \\
\hline $\mathrm{pBAD} / \mathrm{Myc}-\mathrm{His}$ & Amp, ColE1 derivative cloning vector, pBAD (ara) promoter & Invitrogen \\
\hline pBAD-nlpE & pBAD/Myc-His expressing E. coli K12 NlpE-His from the pBAD (ara) promoter & This study \\
\hline $\mathrm{pRCL}$ & $\mathrm{Cm}$, promoterless lac $Z$ & This study \\
\hline $\mathrm{pRCL}-\mathrm{P}_{h \subset p 2 B}$ & pRCL harboring hcp2B promoter & This study \\
\hline pKD46 & Amp, expresses $\lambda$ red recombinase & {$[45]$} \\
\hline pKD3 & $\mathrm{Cm}$, template plasmid & [45] \\
\hline pCP20 & $\mathrm{Cm}$, Amp, yeast Flp recombinase gene, FLP & [45] \\
\hline
\end{tabular}

\section{Bacterial adhesion and invasion assays}

Bacterial adhesion and invasion assays were performed as described previously $[14,47]$. Chicken embryo fibroblast DF-1 cell monolayers were washed with Dulbecco's modified Eagle's medium (DMEM) without fetal bovine serum (Gibco, Grand Island, NY, USA) and infected with bacteria at a multiplicity of infection of 100 for $2 \mathrm{~h}$ at $37{ }^{\circ} \mathrm{C}$ under $5 \% \mathrm{CO}_{2}$. After being washed with PBS, the cells were lysed with $0.5 \%$ Triton X-100, and the bacteria were counted by plating on LB agar plates. For invasion assays, the extracellular adherent bacteria were killed with DMEM containing $100 \mu \mathrm{g} / \mathrm{mL}$ gentamicin for $1 \mathrm{~h}$, then washed and lysed with $0.5 \%$ Triton X-100 to enumerate the invasive bacteria.

\section{Bacterial competition assays in vitro}

Bacterial competition assays were performed as described previously [11, 48] with some modifications. In brief, fresh donor and recipient strains were adjusted to an $\mathrm{OD}_{600 \mathrm{~nm}}$ of 0.5 and mixed at a 5:1 ratio. This mixture was spotted on LB low-salt plates with nitrocellulose membranes for $6 \mathrm{~h}$ at $30{ }^{\circ} \mathrm{C}$. Bacterial spots were collected, diluted and spotted onto LB plates with or without antibiotics for the selection of donor or recipient strains. Then the competition outcomes were calculated as the ratio of the donor strain to the recipient strain.

Quantitative real-time reverse transcription PCR (qRT-PCR) The expression of genes was investigated with qRT-PCR, as described previously $[14,46]$. Briefly, total RNA was extracted from bacteria with TRIzol reagent (Invitrogen), and residual genomic DNA was removed with a Turbo DNase kit (Life Technologies, Carlsbad, CA, USA). cDNA synthesis was performed with a PrimeScript RT reagent kit (TaKaRa) according to the manufacturer's protocol. qRT-PCR was conducted with SYBR Premix Ex Taq (TaKaRa) and gene-specific primers (Table 2), and the data were normalized to the expression of the housekeeping gene $d n a E$. The relative fold change was calculated via the $\triangle \triangle C T$ method [49].

\section{Antibody production and Western blotting}

Polyclonal anti-Hcp2B serum was raised in New Zealand white rabbits through subcutaneous immunization, as described previously [14]. For Western blotting, bacterial samples were subjected to sodium dodecyl sulfate-polyacrylamide gel electrophoresis and transferred 
Table 2 Primers used in this study

\begin{tabular}{|c|c|c|}
\hline Primers & Sequence $\left(5^{\prime} \text { to } 3^{\prime}\right)^{\mathrm{a}}$ & Target genes \\
\hline \multicolumn{3}{|c|}{ For gene expression, deletion and complementation } \\
\hline cpxREx-F & ACGGGATCCATGAATAAAATCCTGTTAGTTGATGA & $c p \times R$ \\
\hline cpxREx-R & ACCAAGCTTTGAAGCAGAAACCATCAGATAG & $c p \times R$ \\
\hline cpxRMu-F & $\begin{array}{l}\text { GGATTAGCGACGTCTGATGACGTAATTTCTGCCTCGGAGGTATTTAAACAG } \\
\text { TGTAGGCTGGAGCTGCTTC }\end{array}$ & Upstream region of $c p x R$ \\
\hline cpxRMu-R & $\begin{array}{l}\text { AAGATGCGCGCGGTTAAGCTGCCTATCATGAAGCAGAAACCATCAGAT } \\
\text { AGCATATGAATATCCTCCTTAG }\end{array}$ & Downstream region of $c p x R$ \\
\hline cpxR-F & TTGATCTTCTGGACGACAGCA & $c p \times R$ \\
\hline$c p \times R-R$ & CTCAGTACCGGTTAACTCCAGT & $c p \times R$ \\
\hline cpxRCo-F & TCCGGATCCATATCAATAATTTCTTGCCGTTC & Upstream region of $c p x R$ \\
\hline cpxRCo-R & CCCAAGCTTGGCCTGACCAATAAAGTTACG & Downstream region of $c p x R$ \\
\hline nlpE-F & GAGCTCGAGGGTGAAAAAAGCGATAGTGA & $n / p E$ \\
\hline nlpE-R & TTCGAATTCTGCCCCAAACTACTGCAATC & $n l p E$ \\
\hline \multicolumn{3}{|l|}{ For RT-qPCR } \\
\hline dnaE RT-F & ATGTCGGAGGCGTAAGGCT & dnaE \\
\hline dnaE RT-R & TCCAGGGCGTCAGTAAACAA & $d n a E$ \\
\hline hсp2B RT-F & GTGAAATGCTGCCGAAAGTG & hср2B \\
\hline hcp2B RT-R & ACAATCGTCGCGTCAGTAAG & $h с p 2 B$ \\
\hline $\operatorname{vgrG~RT-F}$ & CGAAGACGCAGATGACGATAC & $\operatorname{vgrG}$ \\
\hline $\operatorname{vgrG}$ RT-R & GCGTGGATATAGACCTGTTCAC & $\operatorname{vgrG}$ \\
\hline xmtU RT-F & GGTGTCATATCCGGTACATCTC & $x m+U$ \\
\hline$x m t U$ RT-R & CTGAACCATGATAAGCAACAGG & $x m t U$ \\
\hline vipA RT-F & TAACACGCCGTTGGATGAG & vipA \\
\hline vipA RT-R & GTTCAGCCGGAACAACAAAC & vipA \\
\hline clpV RT-F & GAGACGCTCGCTACCATTATT & $c l p V$ \\
\hline clpV RT-R & TGATTTCGTCCGTCACTTCC & $c l p V$ \\
\hline hcp2A RT-F & ACGAAACCGGTGGACAAA & hср2A \\
\hline hcp2A RT-R & GGTTGGTGCGGTAGAATACA & hср2A \\
\hline \multicolumn{3}{|l|}{ For lacZ fusion and EMSA } \\
\hline Phcp2B-F & TCTAAGCTTAGCTTATGTAATCGTGTTCTGAA & Upstream region of $h c p 2 B$ \\
\hline Phcp2B-R & GACGGATCCTTGAAATGTAACATGGGGTTGG & hср2B \\
\hline Phcp2BEMSA-F & AGCTTATGTAATCGTGTTCTG & Upstream region of $h c p 2 B$ \\
\hline Phcp2B EMSA-R & CATGGGGTTGGCATTTATGAA & hср2B \\
\hline Phcp2BdeletionEMSA-F & TTGACTAAAAATATATTTAAAC & Upstream region of $h c p 2 B$ \\
\hline
\end{tabular}

a Restriction sites are underlined.

onto a polyvinylidene fluoride membrane (Amersham Pharmacia Biotech, Piscataway, NJ, USA) as described previously [14, 47]. The proteins were reacted with the primary antibodies anti-Hcp2B or anti-DnaK (Enzo Life Sciences, Farmingdale, NY, USA), followed by horseradish peroxidase conjugated goat anti-mouse or goat antirabbit IgG secondary antibodies. The antigen-antibody complexes were visualized with chemiluminescence substrate (Amersham Pharmacia Biotech).

\section{$\beta$-Galactosidase assays}

To confirm the promoter activity of the $h c p 2 B$ gene, we performed $\beta$-galactosidase assays as described previously
[50]. Briefly, the $\mathrm{P}_{h c p 2 B}-l a c Z$ fusions were constructed by cloning the $\mathrm{P}_{h c p 2 B}$ promoter into the promoterless plasmid pRCL. Then, the bacteria containing the $\mathrm{P}_{h c p 2 B}$-lacZ fusion plasmid or promoterless plasmid $\mathrm{pRCL}$ were collected and resuspended in $\mathrm{Z}$ buffer. The $\beta$-galactosidase activity was quantified with ortho-nitrophenyl- $\beta$ galactoside as the substrate. This assay was performed three times in triplicate.

\section{Electrophoretic mobility shift assay (EMSA)}

The $c p x R$ gene was cloned into the pET28a $(+)$ plasmid (Novagen, Madison, WI, USA), and the recombinant proteins were expressed in E. coli BL21 (DE3) cells by 
addition of $1 \mathrm{mM}$ isopropyl- $\beta$-D-thiogalactopyranoside. The purification of CpxR fusion protein was performed with a HisTrap high-performance column (GE Healthcare, Little Chalfont, Buckinghamshire, UK) as previously described [14]. The CpxR protein was phosphorylated with acetyl phosphate (Sigma, St. Louis, MO, USA) as previously described [51]. Then, EMSA were performed to determine the binding of phosphorylated CpxR (CpxR-P) to the $h c p 2 B$ promoter. Briefly, the sequence of the $h c p 2 B$ promoter region with or without the putative CpxR binding site was amplified and labeled with biotin. The biotin-labeled DNA probe (40 ng) was incubated with increasing concentrations of CpxR-P protein in EMSA binding buffer $(10 \mathrm{mM}$ Tris, $50 \mathrm{mM} \mathrm{KCl}$, $5 \mathrm{mM} \mathrm{MgCl}, 1 \mathrm{mM}$ dithiothreitol, $0.1 \mathrm{mM} \mathrm{MnCl}_{2}$, $2.5 \%$ glycerol and $50 \mathrm{ng} / \mu \mathrm{L}$ poly $[\mathrm{dI}-\mathrm{dC}])$. After incubation for $30 \mathrm{~min}$ at room temperature, the reactions were subjected to electrophoresis and transferred to a nylon membrane. The biotin-labeled DNA was detected with a chemiluminescent substrate (Amersham Pharmacia Biotech). A competitive EMSA was performed by simultaneously incubating the biotin-labeled and unlabeled $h c p 2 B$ promoter region with CpxR-P protein.

\section{Statistical analyses}

Statistical analyses were conducted with the GraphPad Software package. One-way analysis of variance (ANOVA) was used to analyze the results of the adhesion, invasion and bacterial competition assays. Two-way ANOVA was used to analyze the qRT-PCR data. Analysis of the animal infection study results was performed with the non-parametric Mann-Whitney U-test. $P<0.05$ was considered statistically significant.

\section{Results}

\section{Deletion of $c p x R$ attenuates APEC virulence in ducks}

The Cpx TCS has varying effects on the pathogenesis of different bacteria [26-35, 41, 44, 52, 53]. In APEC, the Cpx system has been implicated in the regulation of type 1 fimbriae and found to contribute to adherence, invasiveness and biofilm formation [41]. However, there is limited direct evidence that the CpxR affects the virulence of APEC in vivo. Thus, we constructed and characterized the Cpx TCS regulator encoding gene $c p x R$ mutant and complemented strains. No significant differences were observed in the growth rate or the halo diameter between the wild-type and mutant strains. Then we compared the virulence of these APEC strains in a 7-day-old duck systemic infection model. The results showed that the mortality of DE719, $\Delta$ cpxR and C $\Delta$ cpxR was $75 \%$ (6/8), $25 \%$ $(2 / 8)$ and $62.5 \%(5 / 8)$, respectively. Moreover, the ducks infected with the mutant strain $\Delta$ cpxR died later than those infected with wild-type and complemented strains
(Figure 1A). These results indicated that $\mathrm{CpxR}$ is required for full virulence APEC in ducks.

\section{CpxR provides the colonization and competition fitness for APEC during infection in vivo}

To determine whether the decreased mortality was associated with altered bacterial colonization and survival capacity, we investigated the bacterial loads of APEC strains in the lung, liver and spleen at $24 \mathrm{~h}$ post-infection. The CFUs of the mutant strain $\Delta$ cpxR recovered from the lung, liver and spleen were significantly less than those of the wild-type and complemented strains in the noncompetitive assays $(P<0.05$; Figures $1 \mathrm{~B}-\mathrm{D})$. Bacterial pathogens compete with other bacteria, thus facilitating their replication and survival, and systemic infection [54]. Thus, the effects of CpxR on interbacterial competition fitness and pathogenesis in vivo were further examined by inoculation with equal amounts of mutant and wild-type strains. Similarly, the mutant strain $\Delta \mathrm{cpxR}$ was strongly outcompeted by the wild-type strain in the tested organs (Figure 1E). These results indicated that CpxR potentiates the effective colonization and increases the competition fitness of APEC during infection in vivo.

\section{The role of CpxR in the invasion of APEC to DF-1 cells}

Bacterial adherence to and invasion of host cells are essential for effective colonization and pathogenesis. Moreover, CpxR is involved in the regulation of essential colonization and virulence factors. Therefore, we investigated the influence of CpxR on the bacterial adhesion to and invasion of DF-1 cells. Though the mutant strain $\Delta$ cpxR showed a slightly increased ability to adhere to DF-1 cells, no significant difference was found among these APEC strains (Figure 2A), in agreement with observations from a previous study [41]. However, the mutant strain showed a significantly lower ability than that of the wild-type strain $(P<0.001)$ to invade DF-1 cells. Furthermore, the defect in invasion capacity was restored via trans-complementation with the $c p x R$ gene in the mutant strain (Figure 2B). These results suggested that CpxR contributes to the invasion of APEC to DF-1 cells.

\section{CpxR facilitates the interbacterial competition of APEC in vitro}

To further investigate the role of CpxR in interbacterial competition, we co-cultured fresh APEC donor and recipient strains and counted the surviving bacteria. The survival of the recipient mutant strain $\Delta$ cpxR was significantly decreased when mixed with donor wildtype and complemented strains $(P<0.05)$. In contrast, the donor mutant strain $\Delta$ cpxR could not effectively kill the recipient wild-type and complemented strains (Figure $3 \mathrm{~A})$. Moreover, the mutant strain $\Delta \mathrm{cpxR}$ exhibited 

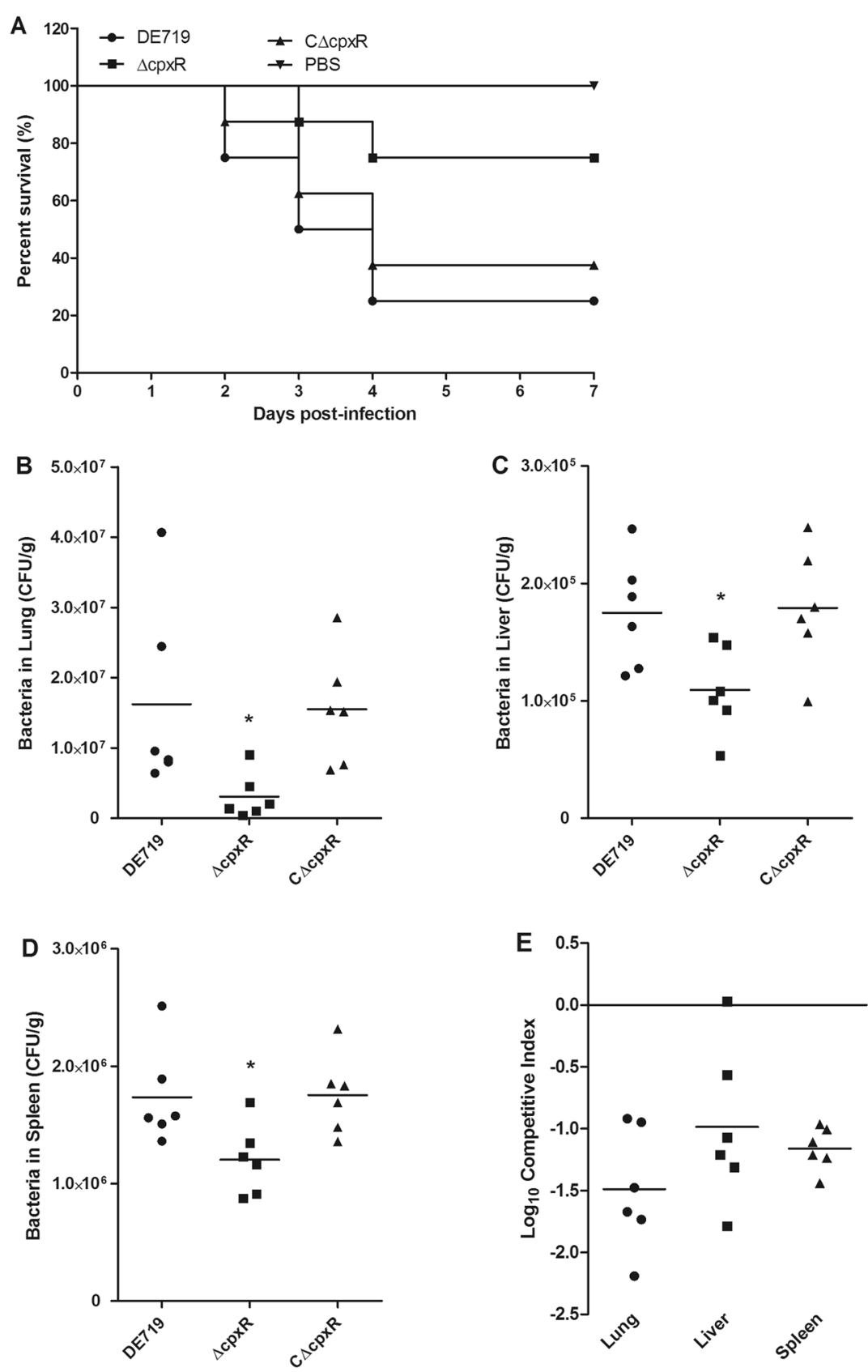

Figure 1 CpxR is essential for efficient colonization and virulence of APEC. A Determination of bacterial virulence. Seven-day-old ducks were infected with APEC strains, and the mortality was monitored until 7 days post-infection. Negative controls were injected with PBS. B-E Bacterial colonization, survival and competition in ducks. Seven-day-old ducks were infected with APEC strains in noncompetitive (B-D) and competitive (E) assays. Ducks were sacrificed at $24 \mathrm{~h}$ post-infection, and bacteria were recovered from the lungs, livers and spleens. The competitive indices were calculated and shown for the competitive assays (E). Nonparametric Mann-Whitney U-test was carried out to determine statistical significance $(* P<0.05)$.

growth comparable to that of the wild-type strain, thus indicating that the decreased interbacterial competition activity of the mutant strain $\Delta c p x R$ was unlikely to be due to a general growth defect. T6SSs have been found to confer a competitive advantage to bacteria, through their interbacterial activity. Previous studies by our group and others have indicated that APEC T6SS2 contributes to the invasion, survival, interbacterial competition and pathogenesis of APEC and NMEC $[11,14-16,55]$. Thus, a competition assay between the 

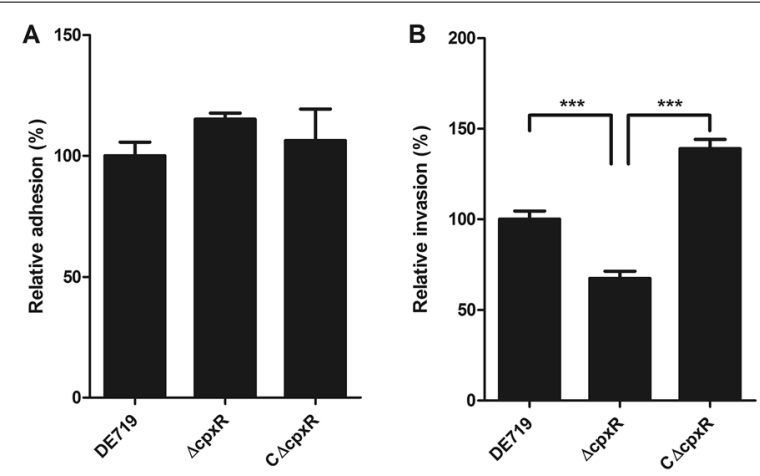

Figure 2 CpxR contributes to the invasion of APEC to DF-1 cells. The ability of APEC strains to adhere to (A) and invade into (B) DF-1 cells was compared. The mutant strain $\triangle c p x R$ showed significantly higher DF-1 cells invasion than the wild-type and complemented strains. Results are shown as relative adhesion and invasion capacity compared with those of the wild-type strain. Error bars indicate standard deviations. Statistical significance analysis was performed by using one-way ANOVA $(* * *<0.001)$.

mutant strains $\Delta \mathrm{T} 6 \mathrm{SS} 2$ and $\Delta \mathrm{cpxR}$ was performed. As expected, the strain $\Delta \mathrm{T} 6 \mathrm{SS} 2$ was outcompeted by the strain $\Delta$ cpxR, suggesting that the deletion of T6SS2 resulted in abolished interbacterial competition activity (Figure 3B).

Previous studies have indicated that overproduction of the outer membrane lipoprotein NlpE upregulates the Cpx pathway in E. coli $[21,22]$. Thus, to further demonstrate the roles of CpxR in interbacterial competition, we overexpressed NlpE in wild-type and mutant strains, and measured the interbacterial competition activity of these strains. As expected, the overexpression of NlpE significantly increased the interbacterial competition activity in the wild-type strain $(P<0.01)$. However, no difference in interbacterial competition activity was observed for the mutant strain $\Delta$ cpxR overexpressing NlpE (Figure 3C). Collectively, these observations indicated that activation of $\mathrm{CpxR}$ facilitates the interbacterial competition activity of APEC.

\section{CpxR regulates the expression of T6SS2 in APEC}

It has been shown that the Cpx TCS plays roles in regulating the T6SS in Citrobacter rodentium [36]. Hence, we sought to determine whether the decreased interbacterial activity of the mutant strain $\Delta c p x R$ was due to changes in T6SS2 expression. The expression of the T6SS2 core genes in these APEC strains was analyzed by qRT-PCR. The results showed that deletion of $c p x R$ significantly downregulated the transcription of the $h c p 2 B$ operon genes $h c p 2 B, \operatorname{vgr} G$ and $x m t U(P<0.001)$. However, the transcript levels of other T6SS core genes vipA, $c l p V$ and $h c p 2 A$ changed only slightly (less than twofold) in the mutant strain $\Delta$ cpxR (Figure 4A), possibly because the vip $A$ and $h c p 2 B$ operons were transcribed in the opposite direction [11]. Additionally, we further validated the levels of Hcp2B via Western blotting. In agreement with the qRT-PCR results, Hcp2B production was decreased in the mutant strain $\triangle \mathrm{cpxR}$ (Figure 4B). Moreover, complementation of the $c p x R$ gene restored the transcription of T6SS2 genes (Figure 4A) and Hcp2B production (Figure 4B).

To further demonstrate that activation of CpxR promotes T6SS2 expression, we overexpressed NlpE in wild-type and mutant strains. The overexpression of NlpE significantly upregulated the transcription of $h c p 2 B, v g r G$ and $x m t U$ genes in the wild-type strain
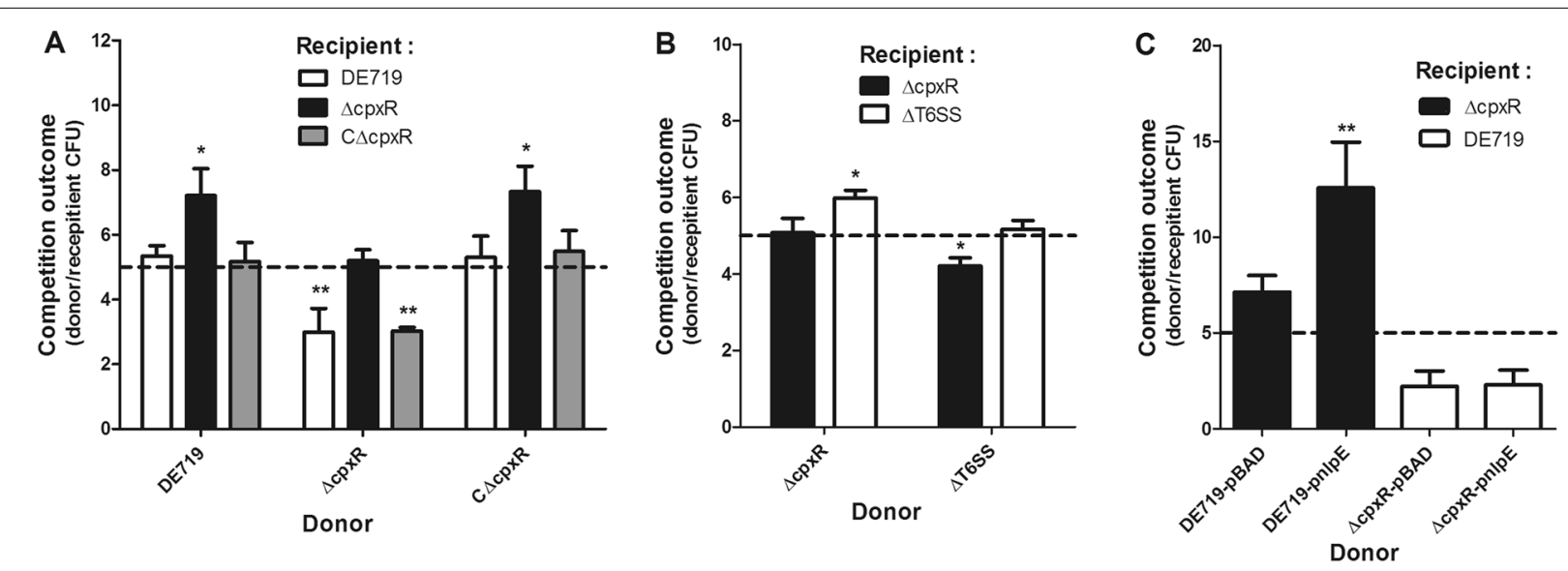

Figure 3 CpxR facilitates interbacterial competition of APEC in vitro. The indicated APEC donor and recipient strains were co-incubated at a ratio of 5:1, as shown by the dashed line. After incubation for $6 \mathrm{~h}$ at $30^{\circ} \mathrm{C}$, the donor and recipient strains were recovered. The competition outcomes between donor and recipient strains were calculated and are shown. A, B The interbacterial competition activities of wild-type, mutant and complemented strains were compared. $\mathbf{C}$ The interbacterial competition activity was also measured in the wild-type and mutant strains with NIpE overexpression. Statistical significance was assessed by using one-way ANOVA. $(* P<0.05 ; * * P<0.01)$. 

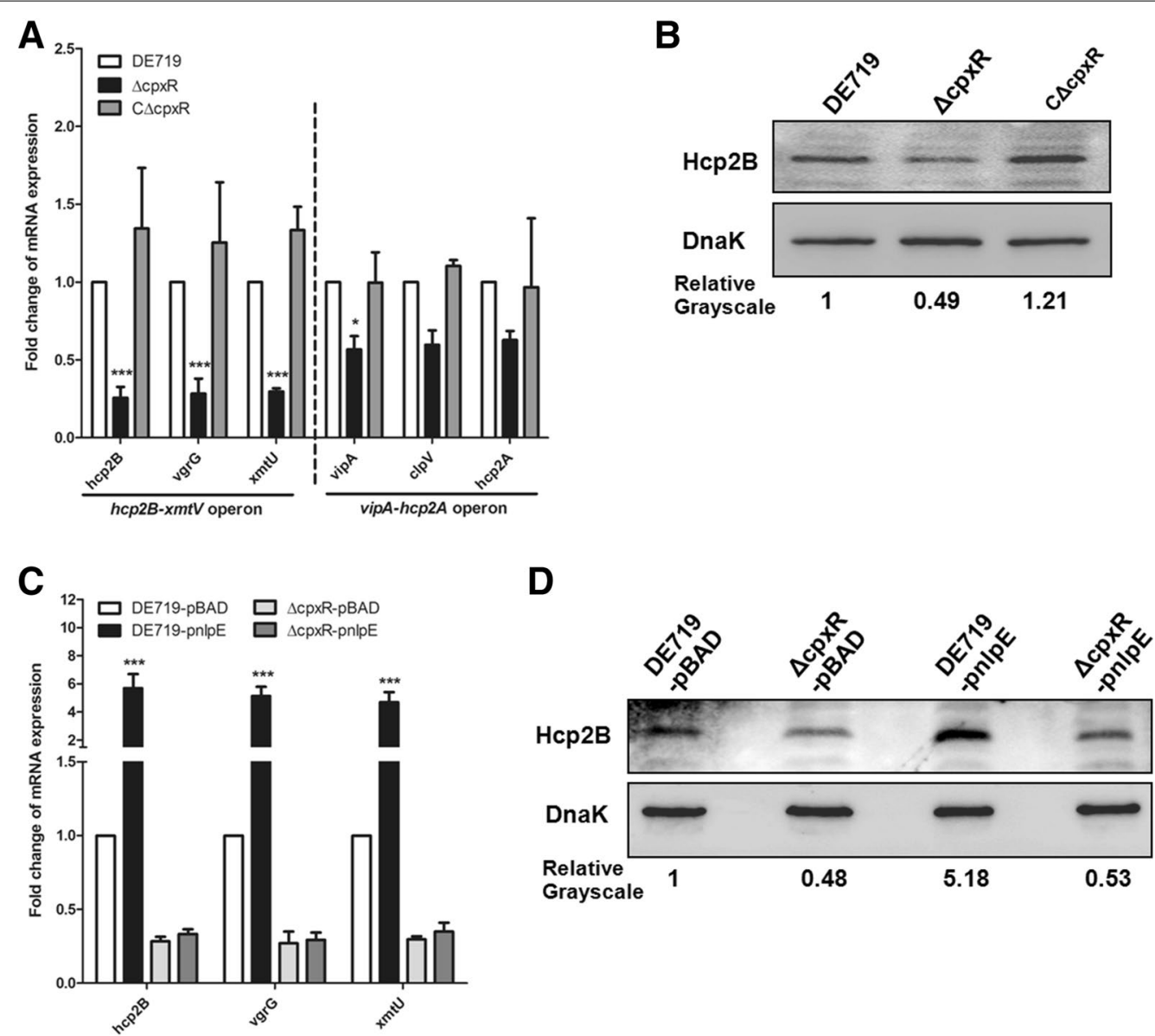

Figure 4 CpxR regulates the expression of T6SS2 genes in APEC. A The transcription levels of T6SS2 core genes were analyzed by qRT-PCR. B The levels of Hcp2B in APEC strains were confirmed with Western blotting. C qRT-PCR analysis for the transcriptional levels of the T6SS2 genes $h c p 2 B, v g r G$ and $x m t U$ in the absence or presence of $\mathrm{NIpE}$ in the wild-type and mutant strains. D The levels of Hcp2B in APEC strains with or without NIpE overexpression were determined by Western blotting. The qRT-PCR data are shown as relative expression ratios compared with that of the wild-type strain. For Western blotting, anti-DnaK antibody was used as a control. The expression of the Hcp2B protein was determined by quantifying the grayscale in Image J software. Two-way ANOVA was carried out to determine statistical significance $\left({ }^{*} P<0.05 ;{ }^{* * *} P<0.001\right)$.

$(P<0.001)$ but not the mutant strain $\Delta$ cpxR (Figure 4 C). Similarly, Hcp2B production increased with CpxR activation in the wild-type strain compared with the mutant strain $\Delta$ cpxR (Figure $4 \mathrm{D}$ ). These data indicated that $\mathrm{CpxR}$ positively regulated the expression of T6SS2 hcp $2 B$ operon in APEC.

\section{CpxR protein directly binds the $h c p 2 B$ promoter region}

To investigate whether CpxR directly regulates $h c p 2 B$ operon expression, we first searched for a putative $C p x R$ binding site in the promoter sequence of the $h c p 2 B$ gene according to the consensus sequence $\mathrm{CpxR}$ binding site [GTAAA $(\mathrm{N})_{4-8}$ GTAAA] reported in E. coli $[56,57]$. One putative CpxR binding site was found to be located in the promoter region of the $h c p 2 B$ gene (Figure $5 \mathrm{~A}$ ), thus suggesting that CpxR might directly regulate the $h c p 2 B$ operon. Furthermore, we demonstrated that the $\operatorname{lac} Z$ transcriptional reporter fusion $\mathrm{P}_{h c p 2 B}$-lac $Z$ under the control of the $h c p 2 B$ promoter showed $\beta$-galactosidase activity (Additional file 1 ). Next, the $h c p 2 B$ promoter sequence including the potential CpxR binding site was amplified, biotin-labeled and subjected to EMSA analysis, to verify the direct binding of CpxR-P to the $h c p 2 B$ promoter region. Indeed, the migration of the $h c p 2 B$ probe was slowed in the presence of increasing amounts of CpxR-P. In addition, binding specificity was confirmed via a competitive EMSA, and the binding of CpxR-P to biotin-labeled probes was abolished by an excess of specific competitor consisting of unlabeled $h c p 2 B$ promoter fragments. In addition, the $h c p 2 B$ promoter fragments without the $C p x R$ binding site were no longer shifted by the CpxR-P protein (Figure 5B). Taken together, these results suggested that $\mathrm{CpxR}$ directly binds the T6SS2 $h c p 2 B$ operon in APEC. 


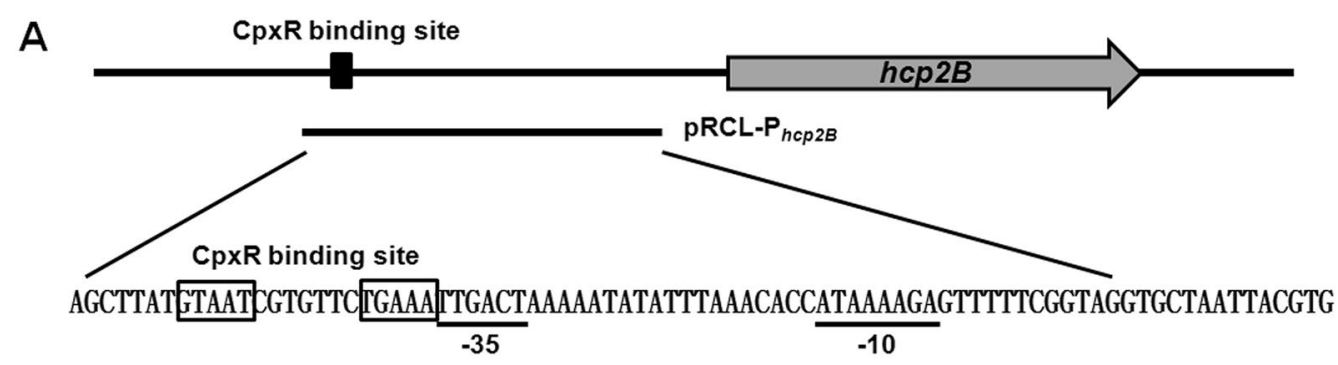

TGCCTGTAACTCGAATAAAGAAAAGGGTGGGTTCCTGATGTGAGCCGCATCACACTCTAACCTGTCGGGGATTAACGACGGG

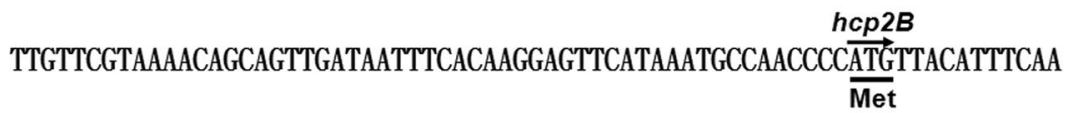

B

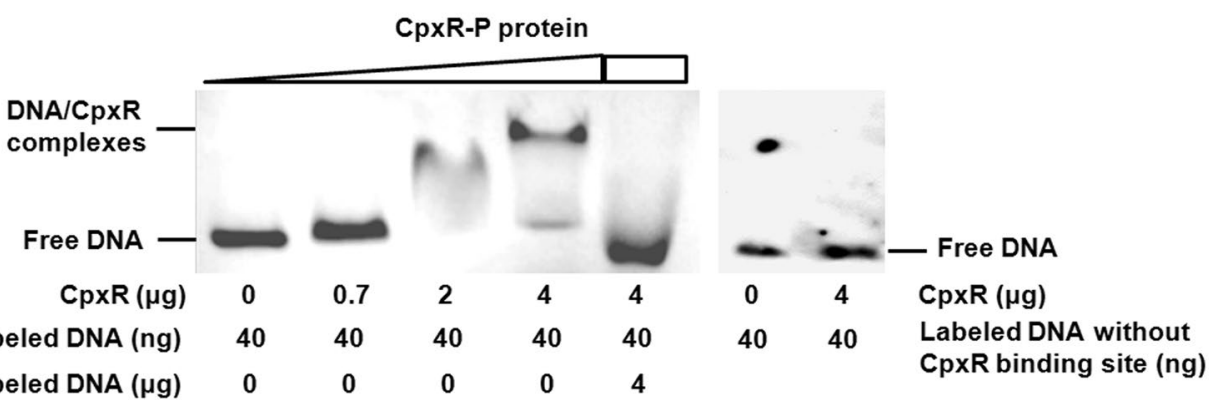

Figure 5 CpxR directly binds the T6SS2 hcp2B promoter region of APEC. A The sequence and schematic representation of the $h c p 2 B$ promoter region. The bold line shows the sequence used in the lacZ fusion and EMSA analyses. The putative CpxR binding site is indicated with boxes. The putative -35 and -10 elements of the promoter are indicated with underlining. B EMSA for the binding of CpxR-P protein to the $h c p 2 B$ promoter region. The hсp2B promoter DNA fragment with or without the CpxR binding site was amplified and biotin-labeled, and the biotin-labeled probe was mixed with increasing amounts of CpxR-P protein. For the specific competitive EMSA, CpxR-P protein was incubated with both the biotin-labeled and the unlabeled DNA probes. The biotin-labeled DNA was detected with a chemiluminescent substrate. The concentrations of CpxR-P protein and probes are shown below the figure.

\section{Discussion}

Bacterial virulence is determined by the expression of virulence factors, and pathogens must be able to precisely modulate gene expression to facilitate their adaptation and survival in the local microenvironment or host cells. The infection process requires rapid adaptation to the host environment through various regulatory mechanisms. TCSs are activated in response to changing environmental cues, thus representing one mechanism enabling bacterial adaptation through transcriptional regulation of gene expression [18, 19]. The Cpx TCS responds to cell envelope stress, which is used by bacteria to maintain cell envelope integrity [23, 58]. Increasing studies implicate the Cpx TCS in the virulence of numerous Gram-negative bacteria [26-35, 41, 44, 52, 53]. These findings prompted us to determine the regulatory roles of the Cpx TCS in the fitness and virulence of APEC. This study provided evidence that the regulator CpxR contributes to the interbacterial competition activity, survival and virulence of APEC at least through directly regulating the expression and function of T6SS2.
By using a duck systemic infection model, we demonstrated that inactivation of the $c p x R$ gene attenuates the virulence of APEC. Moreover, the complemented strain showed recovered virulence. Thus, we concluded that CpxR is necessary for full virulence of APEC. APEC initially infects poultry via the respiratory tract, then spreads systemically throughout the entire body, thus resulting in subsequent bacteremia and death. Colonization and invasion are important virulence parameters in APEC infection $[6,59]$. A previous study has indicated that the Cpx TCS affects virulence features including the adherence, invasiveness, motility and biofilm formation of APEC through the direct binding of CpxR-P to the fimA promoter [41]. Indeed, type 1 fimbriae are filamentous surface organelles known to contribute to adherence, invasiveness and biofilm formation [6, 60]. Our results indicated that inactivation of $\mathrm{CpxR}$ does not affect the adherence ability of APEC, in agreement with findings from a previous study. This observation might have been because CpxR affects the ON/OFF orientation on 
the fimA promoter [41]. In contrast, the mutant strain $\Delta$ cpxR showed a significantly decreased ability to invade into host cells. The decreased capacity of the mutant strain to invade DF-1 cells might contribute to the inability of this mutant to effectively colonize, survive in and infect ducks. Similarly, decreased colonization and virulence have been observed for the $c p x$ mutant strain in different Gram-negative pathogens [31, 61-63].

Pathogens have developed diverse attack strategies to efficiently compete with other bacteria for limited resources and facilitate survival and infection [13]. Our results showed that the interbacterial competition capacity of the mutant strain $\Delta$ cpxR was much lower than that of the wild-type strain in vitro. Moreover, the mutant strain $\Delta$ cpxR was significantly outcompeted by the wildtype strain in ducks. These results indicated that CpxR contributes to competition and survival in vivo, and might be responsible for the systemic infection and virulence of APEC. Consistently with these results, CpxRA influences the initial colonization and outgrowth of Xenorhabdus nematophila during infection through regulation of the nil locus [62]. In addition, the Cpx system may affect bacterial virulence by modulating surface characteristics and serum resistance of pathogens [61].

The E. coli CpxR regulon contains hundreds of genes, according to genome-wide screens for the putative CpxR binding sequence $[56,57]$. In addition to regulating protein folding and degradation, CpxR also plays roles in regulating T6SS expression in Citrobacter rodentium [36]. T6SSs are a common strategy used by many pathogens to mediate successful infections in hosts $[7-14,16]$. The APEC T6SS2 functions in the invasion, survival, interbacterial competition and pathogenesis of APEC and NMEC [11, 14-16, 55]. In this study, we observed that CpxR influenced the expression of T6SS2; we then sought to investigate the mechanism of $\mathrm{CpxR}$ mediated T6SS2 regulation in APEC. We found that inactivation of the regulator CpxR significantly downregulated the transcription of the T6SS2 hcp $2 B$ operon. Although the fold-change in the Hcp2B protein was less than the changes at the transcription level, it was also decreased in the mutant strain $\Delta \mathrm{cpxR}$. Previous study showed that the $h c p 2 B$ operon is necessary for the interbacterial competition activity of T6SS2 in APEC [11]. Moreover, our results showed that the decreased expression of Нcp2B protein was in good agreement with the interbacterial competition activity for mutant strain $\Delta$ cpxR. Our previous study has also found different fold-changes in the transcription and expression levels [64], possibly because many factors affect protein expression. Collectively, these results suggested that CpxR promotes the expression of APEC T6SS2. This result is consistent with the regulatory role in Citrobacter rodentium [36]. Additional, the decreased expression of the T6SS2 hcp $2 B$ operon, including the interbacterial effector encoding the gene $x m t U$, might be responsible for the decreased invasion, diminished survival and impaired competition of the mutant strain $\Delta$ cpxR. Many environmental conditions upregulate the Cpx pathway. The lipoprotein NlpE has been shown to activate the Cpx pathway when it is overproduced [21-23, 58, 65, 66]. We observed that NlpE overexpression in the wild-type strain DE719 increased the T6SS2 $h c p 2 B$ operon expression and subsequently significantly enhanced interbacterial competition. In contrast, no regulatory effect was found for the mutant strain $\Delta c p x R$ overexpressing NlpE. An in silico analysis identified a putative CpxR binding site in the $h c p 2 B$ promoter region. Furthermore, we provided evidence that CpxR-P binds the promoter region of the $h c p 2 B$ operon, thus suggesting that CpxR directly regulates T6SS $2 h c p 2 B$ expression. Taken together, these results indicated that the regulation of T6SS2 expression and function by CpxR may contribute to the infection and pathogenesis of APEC.

In conclusion, we provided evidence that $\mathrm{CpxR}$ contributes to colonization, invasion and interbacterial competition fitness for APEC in vivo and in vitro. The most provocative finding of this study is that CpxR may contribute to APEC virulence by directly binding and regulating the expression and function of T6SS2 hcp $2 B$ (Figure 6). Together with the conclusion that the $\mathrm{Cpx}$

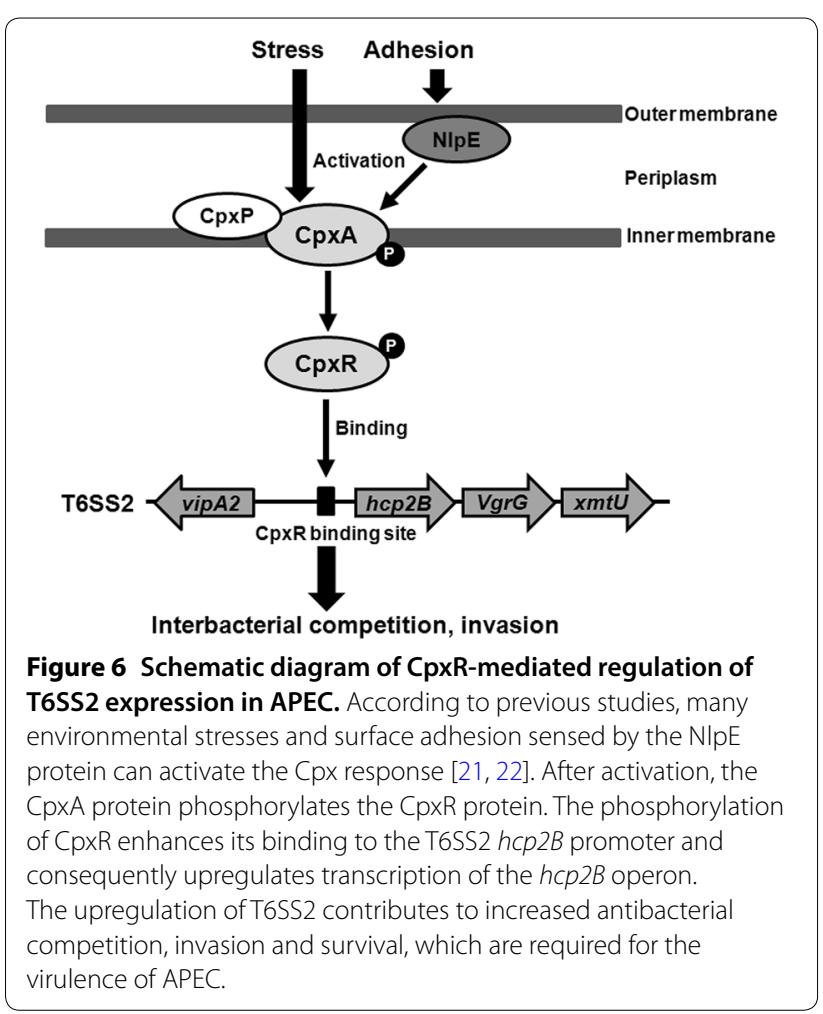


TCS regulates type 1 fimbriae-mediated virulence features, including adherence, motility and biofilm formation of APEC [41], these compelling findings suggest that the Cpx TCS controls the virulence of APEC via various regulatory mechanisms. Thus, further studies are needed to fully understand the regulatory network through which Cpx TCS contributes to virulence.

\section{Additional file}

\section{Additional file 1 . The $\beta$-galactosidase activity of the lac $Z$ transcrip- tional reporter fusion $\mathrm{P}_{h c p 2 B}-$ lac $Z$ was measured as described in "Materials and methods".}

\section{Acknowledgements}

We thank the staff of Shanghai Tolo Biotechnology Co. Ltd for helps in Electrophoretic mobility shift assay (EMSA).

\section{Authors' contributions}

SW designed the research studies. ZY, DW, SX and DZ performed the experiments. SW, ZY, DW, TL, MT, JQ, CD and SY analyzed the data and discussed the results. $Z Y, S W$ and SY wrote the manuscript. All authors read and approved the final manuscript.

\section{Funding}

This work was supported by the National Natural Science Foundation of China (Grant Number 31572523) and the National Key Research and Development Program of China (Grant Number 2016YFD0500800). The funders had no role in study design, data collection and analysis, decision to publish or preparation of the manuscript.

\section{Ethics approval and consent to participate}

All animal experiments were conducted in strict accordance with the Guidelines on the Humane Treatment of Laboratory Animals and were approved by the Institutional Animal Care and Use Committee of the Shanghai Veterinary Research Institute (Permit No: Shvri-Po-0080).

\section{Competing interests}

The authors declare that they have no competing interests.

Received: 31 January 2019 Accepted: 8 May 2019

Published online: 24 May 2019

\section{References}

1. Ewers C, Li G, Wilking H, Kiessling S, Alt K, Antao EM, Laturnus C, Diehl I, Glodde S, Homeier T, Bohnke U, Steinruck H, Philipp HC, Wieler LH (2007) Avian pathogenic, uropathogenic, and newborn meningitiscausing Escherichia coli: how closely related are they? Int J Med Microbiol 297:163-176

2. Tivendale KA, Logue CM, Kariyawasam S, Jordan D, Hussein A, Li G, Wannemuehler Y, Nolan LK (2010) Avian-pathogenic Escherichia coli strains are similar to neonatal meningitis E. coli strains and are able to cause meningitis in the rat model of human disease. Infect Immun 78:3412-3419

3. Moulin-Schouleur M, Reperant M, Laurent S, Bree A, Mignon-Grasteau S, Germon P, Rasschaert D, Schouler C (2007) Extraintestinal pathogenic Escherichia coli strains of avian and human origin: link between phylogenetic relationships and common virulence patterns. J Clin Microbiol 45:3366-3376

4. Bauchart P, Germon P, Bree A, Oswald E, Hacker J, Dobrindt U (2010) Pathogenomic comparison of human extraintestinal and avian pathogenic Escherichia coli-search for factors involved in host specificity or zoonotic potential. Microb Pathog 49:105-115
5. Rendon MA, Saldana Z, Erdem AL, Monteiro-Neto V, Vazquez A, Kaper JB, Puente JL, Giron JA (2007) Commensal and pathogenic Escherichia coli use a common pilus adherence factor for epithelial cell colonization. Proc Natl Acad Sci U S A 104:10637-10642

6. Croxen MA, Finlay BB (2010) Molecular mechanisms of Escherichia coli pathogenicity. Nat Rev Microbiol 8:26-38

7. Bernard CS, Brunet YR, Gueguen E, Cascales E (2010) Nooks and crannies in type $\mathrm{VI}$ secretion regulation. J Bacteriol 192:3850-3860

8. Filloux A (2009) The type VI secretion system: a tubular story. EMBO J 28:309-310

9. Hachani A, Wood TE, Filloux A (2016) Type VI secretion and anti-host effectors. Curr Opin Microbiol 29:81-93

10. Jani AJ, Cotter PA (2010) Type VI secretion: not just for pathogenesis anymore. Cell Host Microbe 8:2-6

11. Ma J, Sun M, Pan Z, Song W, Lu C, Yao H (2018) Three Hcp homologs with divergent extended loop regions exhibit different functions in avian pathogenic Escherichia coli. Emerg Microbes Infect 7:49

12. Russell AB, Hood RD, Bui NK, LeRoux M, Vollmer W, Mougous JD (2011) Type VI secretion delivers bacteriolytic effectors to target cells. Nature 475:343-347

13. Sana TG, Flaugnatti N, Lugo KA, Lam LH, Jacobson A, Baylot V, Durand E, Journet L, Cascales E, Monack DM (2016) Salmonella Typhimurium utilizes a T6SS-mediated antibacterial weapon to establish in the host gut. Proc Natl Acad Sci U S A 113:E5044-E5051

14. Wang S, Dai J, Meng Q, Han X, Han Y, Zhao Y, Yang D, Ding C, Yu S (2014) DotU expression is highly induced during in vivo infection and responsible for virulence and Hcp1 secretion in avian pathogenic Escherichia coli. Front Microbiol 5:588

15. Zhou Y, Tao J, Yu H, Ni J, Zeng L, Teng Q, Kim KS, Zhao GP, Guo X, Yao Y (2012) Hcp family proteins secreted via the type VI secretion system coordinately regulate Escherichia coli $\mathrm{K} 1$ interaction with human brain microvascular endothelial cells. Infect Immun 80:1243-1251

16. Ma J, Bao Y, Sun M, Dong W, Pan Z, Zhang W, Lu C, Yao H (2014) Two functional type $\mathrm{VI}$ secretion systems in avian pathogenic Escherichia coli are involved in different pathogenic pathways. Infect Immun 82:3867-3879

17. Silverman JM, Brunet YR, Cascales E, Mougous JD (2012) Structure and regulation of the type $\mathrm{VI}$ secretion system. Annu Rev Microbiol 66:453-472

18. Krell T, Lacal J, Busch A, Silva-Jimenez H, Guazzaroni ME, Ramos JL (2010) Bacterial sensor kinases: diversity in the recognition of environmental signals. Annu Rev Microbiol 64:539-559

19. Capra EJ, Laub MT (2012) Evolution of two-component signal transduction systems. Annu Rev Microbiol 66:325-347

20. MacRitchie DM, Buelow DR, Price NL, Raivio TL (2008) Two-component signaling and gram negative envelope stress response systems. Adv Exp Med Biol 631:80-110

21. Vogt SL, Raivio TL (2012) Just scratching the surface: an expanding view of the Cpx envelope stress response. FEMS Microbiol Lett 326:2-11

22. Snyder WB, Davis LJ, Danese PN, Cosma CL, Silhavy TJ (1995) Overproduction of NIpE, a new outer membrane lipoprotein, suppresses the toxicity of periplasmic LacZ by activation of the Cpx signal transduction pathway. J Bacteriol 177:4216-4223

23. Hunke S, Keller R, Muller VS (2012) Signal integration by the Cpx-envelope stress system. FEMS Microbiol Lett 326:12-22

24. Wolfe AJ, Parikh N, Lima BP, Zemaitaitis B (2008) Signal integration by the two-component signal transduction response regulator CpxR. J Bacteriol 190:2314-2322

25. Raivio TL, Popkin DL, Silhavy TJ (1999) The Cpx envelope stress response is controlled by amplification and feedback inhibition. J Bacteriol 181:5263-5272

26. Herbert EE, Cowles KN, Goodrich-Blair H (2007) CpxRA regulates mutualism and pathogenesis in Xenorhabdus nematophila. Appl Environ Microbiol 73:7826-7836

27. Nevesinjac AZ, Raivio TL (2005) The Cpx envelope stress response affects expression of the type IV bundle-forming pili of enteropathogenic Escherichia coli. J Bacteriol 187:672-686

28. Hung DL, Raivio TL, Jones CH, Silhavy TJ, Hultgren SJ (2001) Cpx signaling pathway monitors biogenesis and affects assembly and expression of $P$ pili. EMBO J 20:1508-1518

29. Weatherspoon-Griffin N, Zhao G, Kong W, Kong Y, Morigen AndrewsPolymenis H, McClelland M, Shi Y (2011) The CpxR/CpxA two-component 
system up-regulates two Tat-dependent peptidoglycan amidases to confer bacterial resistance to antimicrobial peptide. J Biol Chem 286:5529-5539

30. Herbert Tran EE, Goodrich-Blair H (2009) CpxRA contributes to Xenorhabdus nematophila virulence through regulation of IrhA and modulation of insect immunity. Appl Environ Microbiol 75:3998-4006

31. Thomassin JL, Giannakopoulou N, Zhu L, Gross J, Salmon K, Leclerc JM, Daigle F, Le Moual H, Gruenheid S (2015) The CpxRA two-component system is essential for Citrobacter rodentium virulence. Infect Immun 83:1919-1928

32. Nakayama S, Watanabe H (1998) Identification of $c p x R$ as a positive regulator essential for expression of the Shigella sonnei virF gene. J Bacteriol 180:3522-3528

33. Gal-Mor O, Segal G (2003) Identification of CpxR as a positive regulator of icm and dot virulence genes of Legionella pneumophila. J Bacteriol 185:4908-4919

34. Humphreys S, Rowley G, Stevenson A, Anjum MF, Woodward MJ, Gilbert S, Kormanec J, Roberts M (2004) Role of the two-component regulator CpxAR in the virulence of Salmonella enterica serotype Typhimurium. Infect Immun 72:4654-4661

35. De la Cruz MA, Perez-Morales D, Palacios IJ, Fernandez-Mora M, Calva E, Bustamante VH (2015) The two-component system CpxR/A represses the expression of Salmonella virulence genes by affecting the stability of the transcriptional regulator HilD. Front Microbiol 6:807

36. Giannakopoulou N, Mendis N, Zhu L, Gruenheid S, Faucher SP, Le Moual H (2018) The virulence effect of CpxRA in Citrobacter rodentium is independent of the auxiliary proteins NIpE and CpxP. Front Cell Infect Microbiol 8:320

37. Altman E, Segal G (2008) The response regulator CpxR directly regulates expression of several Legionella pneumophila icm/dot components as well as new translocated substrates. J Bacteriol 190:1985-1996

38. O'Loughlin JL, Spinner JL, Minnich SA, Kobayashi SD (2010) Yersinia pestis two-component gene regulatory systems promote survival in human neutrophils. Infect Immun 78:773-782

39. Labandeira-Rey M, Brautigam CA, Hansen EJ (2010) Characterization of the CpxRA regulon in Haemophilus ducreyi. Infect Immun 78:4779-4791

40. Labandeira-Rey M, Dodd D, Fortney KR, Zwickl B, Katz BP, Janowicz DM, Spinola SM, Hansen EJ (2011) A Haemophilus ducreyi CpxR deletion mutant is virulent in human volunteers. J Infect Dis 203:1859-1865

41. Matter LB, Ares MA, Abundes-Gallegos J, Cedillo ML, Yanez JA, MartinezLaguna Y, De la Cruz MA, Giron JA (2018) The CpxRA stress response system regulates virulence features of avian pathogenic Escherichia coli. Environ Microbiol 20:3363-3377

42. De la Cruz MA, Ruiz-Tagle A, Ares MA, Pacheco S, Yanez JA, Cedillo L, Torres J, Giron JA (2017) The expression of Longus type 4 pilus of enterotoxigenic Escherichia coli is regulated by LngR and LngS and by H-NS, CpxR and CRP global regulators. Environ Microbiol 19:1761-1775

43. Dudin O, Geiselmann J, Ogasawara H, Ishihama A, Lacour S (2014) Repression of flagellar genes in exponential phase by CsgD and CpxR, two crucial modulators of Escherichia coli biofilm formation. J Bacteriol 196:707-715

44. De la Cruz MA, Morgan JK, Ares MA, Yanez-Santos JA, Riordan JT, Giron JA (2016) The two-component system CpxRA negatively regulates the locus of enterocyte effacement of enterohemorrhagic Escherichia coli involving sigma(32) and Lon protease. Front Cell Infect Microbiol 6:11

45. Datsenko KA, Wanner BL (2000) One-step inactivation of chromosomal genes in Escherichia coli K-12 using PCR products. Proc Natl Acad Sci U S A 97:6640-6645

46. Wang S, Liu X, Xu X, Yang D, Wang D, Han X, Shi Y, Tian M, Ding C, Peng D, Yu S (2016) Escherichia coli type III secretion system 2 ATPase EivC is involved in the motility and virulence of avian pathogenic Escherichia coli. Front Microbiol 7:1387

47. Wang S, Niu C, Shi Z, Xia Y, Yaqoob M, Dai J, Lu C (2011) Effects of ibeA deletion on virulence and biofilm formation of avian pathogenic Escherichia coli. Infect Immun 79:279-287
48. Brunet YR, Khodr A, Logger L, Aussel L, Mignot T, Rimsky S, Cascales E (2015) H-NS silencing of the Salmonella pathogenicity island 6-encoded type VI secretion system limits Salmonella enterica serovar Typhimurium interbacterial killing. Infect Immun 83:2738-2750

49. Livak KJ, Schmittgen TD (2001) Analysis of relative gene expression data using real-time quantitative PCR and the 2(-Delta Delta C(T)) Method. Methods 25:402-408

50. Cai W, Wannemuehler Y, Dellanna G, Nicholson B, Barbieri NL, Kariyawasam S, Feng Y, Logue CM, Nolan LK, Li G (2013) A novel two-component signaling system facilitates uropathogenic Escherichia coli's ability to exploit abundant host metabolites. PLoS Pathog 9:e1003428

51. Hernday AD, Braaten BA, Broitman-Maduro G, Engelberts P, Low DA (2004) Regulation of the pap epigenetic switch by CpxAR: phosphorylated CpxR inhibits transition to the phase ON state by competition with Lrp. Mol Cell 16:537-547

52. Leuko S, Raivio TL (2012) Mutations that impact the enteropathogenic Escherichia coli Cpx envelope stress response attenuate virulence in Galleria mellonella. Infect Immun 80:3077-3085

53. Mitobe J, Arakawa E, Watanabe H (2005) A sensor of the two-component system CpxA affects expression of the type III secretion system through posttranscriptional processing of InvE. J Bacteriol 187:107-113

54. Coyte KZ, Schluter J, Foster KR (2015) The ecology of the microbiome: networks, competition, and stability. Science 350:663-666

55. Ma J, Sun M, Bao Y, Pan Z, Zhang W, Lu C, Yao H (2013) Genetic diversity and features analysis of type $\mathrm{VI}$ secretion systems loci in avian pathogenic Escherichia coli by wide genomic scanning. Infect Genet Evol 20:454-464

56. Price NL, Raivio TL (2009) Characterization of the Cpx regulon in Escherichia coli strain MC4100. J Bacteriol 191:1798-1815

57. De Wulf P, McGuire AM, Liu X, Lin EC (2002) Genome-wide profiling of promoter recognition by the two-component response regulator CpxR-P in Escherichia coli. J Biol Chem 277:26652-26661

58. DiGiuseppe PA, Silhavy TJ (2003) Signal detection and target gene induction by the CpxRA two-component system. J Bacteriol 185:2432-2440

59. Guabiraba R, Schouler C (2015) Avian colibacillosis: still many black holes. FEMS Microbiol Lett 362:fnv118

60. Pourbakhsh SA, Dho-Moulin M, Bree A, Desautels C, Martineau-Doize B, Fairbrother JM (1997) Localization of the in vivo expression of P and F1 fimbriae in chickens experimentally inoculated with pathogenic Escherichia coli. Microb Pathog 22:331-341

61. Debnath I, Norton JP, Barber AE, Ott EM, Dhakal BK, Kulesus RR, Mulvey MA (2013) The Cpx stress response system potentiates the fitness and virulence of uropathogenic Escherichia coli. Infect Immun 81:1450-1459

62. Herbert Tran EE, Andersen AW, Goodrich-Blair H (2009) CpxRA influences Xenorhabdus nematophila colonization initiation and outgrowth in Steinernema carpocapsae nematodes through regulation of the nil locus. Appl Environ Microbiol 75:4007-4014

63. Li H, Liu F, Peng W, Yan K, Zhao H, Liu T, Cheng H, Chang P, Yuan F, Chen $\mathrm{H}$, Bei W (2018) The CpxA/CpxR two-component system affects biofilm formation and virulence in Actinobacillus pleuropneumoniae. Front Cell Infect Microbiol 8:72

64. Tian M, Qu J, Han X, Ding C, Wang S, Peng D, Yu S (2014) Mechanism of Asp24 upregulation in Brucella abortus rough mutant with a disrupted O-antigen export system and effect of Asp24 in bacterial intracellular survival. Infect Immun 82:2840-2850

65. Otto K, Silhavy TJ (2002) Surface sensing and adhesion of Escherichia coli controlled by the Cpx-signaling pathway. Proc Natl Acad Sci U S A 99:2287-2292

66. Raivio TL, Silhavy TJ (1997) Transduction of envelope stress in Escherichia coli by the Cpx two-component system. J Bacteriol 179:7724-7733

\section{Publisher's Note}

Springer Nature remains neutral with regard to jurisdictional claims in published maps and institutional affiliations. 